\title{
Changes in coronary risk factors during comprehensive five-year community programme to control cardiovascular diseases (North Karelia project)
}

\author{
PEKKA PUSKA, JAAKKO TUOMILEHTO, JUKKA SALONEN, LIISA NEITTAANMÄKI, \\ JUHÄNI MAKI, JARMO VIRTAMO, AULIKKI NISSINEN, KAJ KOSKELA, TUULA TAKALO
}

British Medical fournal, 1979, 2, 1173-1178

\section{Summary and conclusions}

A comprehensive community programme to control cardiovascular diseases (CVD) in North Karelia, Finland, was carried out during 1972-7. The central intermediate objective of the programme was to reduce the prevalence of smoking, the serum cholesterol concentration, and raised blood-pressure values among the population of the area. The effect was evaluated by examining independent representative population samples in 1972 and 1977 in both the county of North Karelia and a matched control county. Over 10000 subjects were studied each time, the participation rate being around $90 \%$. The decrease that occurred in the risk factors, especially in men, was in general greater in North Karelia compared with the control county. When a multiple logistic function was used for the three risk factors an overall mean net reduction of $17 \%$ among men and $12 \%$ among women was observed in the estimated risk for coronary heart disease in North Karelia.

This community programme effectively reduced the levels of the three main risk factors for CVD in the population, and thus mortality and morbidity from CVD should fall. This is assessed in further studies.

Co-ordinating Centre for the North Karelia Project: University of Kuopio, Box 40, 70101 Kuopio 10, Finland, and Epidemiological Research Unit, National Public Health Laboratory, Kuopio PEKKA PUSKA, MD, MA, director of project and of research unit JUKKA SALONEN, MD, special research investigator

JAAKKO TUOMILEHTO, MD, MA, principal investigator

LIISA NEITTAANMÄKI, LICSOCSCI, research investigator

JUHANI MÄKI, MA, systems analyst

JARMO VIRTAMO, MD, research investigator

AULIKKI NISSINEN, MD, NT, research investigator

KAJ KOSKELA, MA, research investigator

TUULA TAKALO, MA, research investigator

\section{Introduction}

In most industrialised countries cardiovascular diseases (CVD) are the main cause of mortality and severe morbidity. Nevertheless, obvious differences exist among countries in the mortality and occurrence of the severe forms of these diseases. The prevalence of coronary heart disease (CHD) seems to be greater in Finland than in any other country. ${ }^{1-4} \mathrm{~W}$ ithin Finland regional differences occur in the mortality and morbidity from CVD, the highest rates being found in the county of North Karelia. ${ }^{15-7}$ Both the mortality from ischaemic heart disease and the regional differences in total mortality increased during the $1960 \mathrm{~s}^{8}{ }^{\circ}$

Because of the exceptionally serious problem in North Karelia, which is a large, mainly rural county in eastern Finland with a population of about 180000 , members of Parliament and other representatives of the local population signed a petition in 1971 asking for national help in reducing the high mortality and morbidity of CVD. The North Karelia project was launched in 1972 for this reason. The project was to consist of a systematic, comprehensive community programme to control CVD in North Karelia and a scientific evaluation of the results obtained.

\section{THE PROGRAMME AND ITS EVALUATION}

At the planning stage it was decided to carry out a comprehensive cardiovascular programme within the structure of the health and social services in the community. This programme aimed at both primary and secondary prevention. ${ }^{1011}$ The objectives of the programme were defined as follows. Main objective: to reduce mortality and morbidity, especially from CVD, with special reference to middle-aged men. Intermediate objectives: to reduce the known cardiovascular risk factorsthat is, smoking, serum cholesterol concentration, and blood pressure-and to promote early detection, treatment, and rehabilitation in people with severe CVD. National objective: to test the feasibility and effect of this approach and to provide tested methods and programmes for nationwide use in connection with the control of CVD and other health problems. The risk factors to be studied were chosen on the basis of information 
from previous studies, collective international recommendations, and epidemiological data from the area.

The baseline information concerning the CVDs and their main risk factors in North Karelia was studied in detail, and some results of the baseline survey, which is described below, have been reported. ${ }^{11}$ They confirm the high general level of the primary risk factors of smoking, cholesterol concentration, and blood pressure. About $52^{\circ}$ of the men in the study (aged 25-59) were current smokers; their mean serum cholesterol concentration was $7.0 \mathrm{mmol} / 1(269 \mathrm{mg} / 100 \mathrm{ml})$ and mean casual blood pressure $147 / 91 \mathrm{~mm} \mathrm{Hg}: 23 \%$ had a systolic blood pressure of at least $160 \mathrm{~mm} \mathrm{Hg}, 34 \%$ a diastolic pressure of at least $95 \mathrm{~mm}$ $\mathrm{Hg}$, and in $19 \%$ both pressures were above these values. Owing to the high general level of the known CHD risk factors and the behavioural and environmental backgrounds of these factors, a community-based programme was found necessary, and the strategy was outlined accordingly. The following principles were especially emphasised. To influence the combined value of the risk factors in the total population and the whole community; to make a "community diagnosis" of the baseline information in order to design the appropriate measures of strategic importance; to carry out a systematic programme and reallocate the existing service resources to control this modern epidemic; to integrate the activities of the existing health and social services in the community and to have the full participation of the community; and to have a continuous follow-up of progress and feedback to the community.

The epidemiological considerations meant that heavy emphasis was laid on the primary prevention of the numerous disease attacks by a mass "community action" against the risk factors. A community programme was designed to lead to the objectives and was gradually implemented systematically. Subprogrammes were planned following the natural course of $\mathrm{CHD}$ and related to smoking, diet, hypertension, CHD, acute myocardial infarction, and rehabilitation. The practical objectives and measures and the recommended inbuilt continuous evaluation were outlined in each subprogramme. Special subprogrammes dealing with screening and the training of local personnel were also set up.

The comprehensive programme was thus integrated into the health and social services of the county. The following elements of the programme were started. (1) Information was given to the public, especially about the practical activities against the risk factors being carried out in the community, by means of newspapers, radio, leaflets, posters, and stickers, and at health education meetings and public campaigns, schools, and places of work. (2) Organisation of services: by systematically integrating the programme into the existing services and creating new services when necessary-for example, making use of basic community health services (health centres), special supporting services (for example, for stopping smoking), and services of other organisations. (3) Training personnel, especially for the practical tasks of the programme. We trained health personnel, special workers, teachers, voluntary workers, and community leaders. (4) Environmental services: these were carried out to support the desired life style-for example, with regard to smoking restrictions, low-fat dairy and meat products, vegetable production, and promotion of sales of health articles in shops. (5) Internal information services: to support the practical activities-for example, by patient cards and files, registers (of hypertension, infarction, and stroke), follow-up surveys, and other information. The intervention has been described elsewhere in greater detail. ${ }^{12}$

The aim of the study was to assess the feasibility and effect of the programme, and to estimate the costs and obtain a comprehensive picture of the changes that took place in the community studied. In assessing the effect of the programme we examined the degree to which the main and intermediate objectives were fulfilled. This included in particular assessing the changes in the risk factors among the middle-aged population brought about by the programme and the resulting changes in mortality and morbidity. We report here the results concerning changes in the risk factors as judged by comparing data obtained in the baseline survey and the five-year terminal survey from the study area and a matched control area. The county of Kuopio, also in eastern Finland, was chosen as the control area because of its close similarity to North Karelia.

\section{Subjects and methods}

The baseline survey was carried out in spring 1972 in North Karelia and the control area. A representative random $6.6^{\circ} \%$ sample was drawn from the population of the two counties by using the national population register. The sample comprised men and women born during 1913-47 (then aged 25-59). Exactly five years later another cross-sectional survey, the five-year terminal survey, was carried out in the two areas. The methods used were strictly the same as those in the baseline survey (see below). The sample was an independent one representing the population at that time: again a $6.6 \%$ random sample was used that comprised men and women born in 1913-47 (now aged 30-64).

Ten days before the examination the subjects received a letter, which contained an explanation of the study, an invitation to the examination, some practical instructions, and a questionnaire. The questionnaire contained 130 mainly precoded questions, most of them pretested on several occasions. Subjects were asked to answer the questionnaire at home and to take it with them to the examination. The questionnaire contained questions on the following groups of items: general background; socioeconomic state; medical history (including present somatic and psychosomatic symptoms) and use of health services; health behaviour (smoking, diet, alcohol consumption, physical exercise) and attempts to change it; attitudes towards risk factors and health services; and social interaction and psychosocial stress.

At the examination height, weight, triceps, skinfold thickness, and blood pressure were measured, and a venous blood specimen was taken to determine serum cholesterol concentrations. Casual blood pressure was measured in a sitting position according to the standardised technique, the fifth phase being recorded as the diastolic pressure..$^{13}$ Subjects were asked to fast for at least four hours before the examination and to avoid any heavy meals during the day of the examination. Serum cholesterol concentrations were determined from deep-frozen samples in a central laboratory in Helsinki, which is standardised against the international references in Atlanta. ${ }^{14} 15$

The questionnaire was checked at the examination, when the subjects were also tested on their knowledge of other aspects of health. Those with known hypertension answered special questions about their treatment. An additional interview about some important aspects of fat consumption was also done by the examination team.

The field work was carried out during February to April, mainly at local health centres, by six groups of two specially trained nurses, each group being aided by two to four local assistants, mostly public health nurses. The trained nurses took blood pressure and skinfold measurements and also checked the questionnaires. Subjects whose blood pressure was high at the examination were told, and their blood pressures were checked afterwards by local health workers. Subjects were informed about their possible high blood lipid concentrations afterwards by letter, which also contained the necessary instructions.

In the analysis those subjects who reported ever having smoked regularly or having smoked during the preceding month and on an average more than once a day were classified as smokers. The reported number of cigarettes, cigars, and pipes smoked per day was calculated as the amount of smoking for each smoker.

In the baseline survey a second invitation and thus the examination of non-participants was not possible; the same questionnaire was, however, posted to the non-participants, whose addresses had been checked. The information obtained in this way was included when data from the questionnaire were analysed. In the terminal survey a second invitation to attend was sent and the examination carried out. Participation, problems, and attitudes concerning the surveys were studied carefully. A separate substudy among a sample of participants in the baseline survey showed that the reactions towards the study were generally favourable. ${ }^{16}$

Table Im shows the sample sizes, participation rates, and reasons for non-participation, while table IIm shows the subjects studied according to age and sex. Because the same cohort was followed up the ages given in the tables refer always to the age in 1972. Some subjects were excluded from the original sample because they had died or moved permanently outside the counties before the survey and thus 
did not belong to the study population. The participation rates were high, being, in the baseline survey, $94^{\circ}{ }_{j}$ in North Karelia and $91 \%$ in the control area (for about $6 \%$ of these subjects only data from the questionnaire were available), and in the terminal survey $89^{\circ} \mathrm{o}$ in North Karelia and $91^{\circ}$ in the control area. The reasons for nonparticipation were mostly unknown: in many cases addresses were not up to date and could not be corrected afterwards. The second largest group of non-participants comprised people temporarily away from home, and the third largest group comprised people unable to answer. Few people refused to participate.

The changes in the risk factors were examined by comparing results obtained in North Karelia and the control area at the start (1972) and end (1977) of the study. The differences between the two counties, and ultimately the changes in the differences during the intervention period (net reduction in North Karelia), were calculated. A $95^{\circ}, 0$ confidence limit was determined for each variable; the tables also show the results of two-tailed tests (Student's $t$ test) of the significance of differences. No adjustment for age was found necessary, because the age distributions in the two areas and at the two times were similar. Each risk factor was initially studied separately, after which a CHD risk estimate was used, based on the three risk factors. In calculating the risk estimate a multiple logistic risk function was used, which was fitted by the least squares iterative procedure of Walker and Duncan. ${ }^{17}$ The coefficients of the risk factors in this model were derived from a prospective five-year follow-up of the cohort in the baseline survey in North Karelia for acute myocardial infarction (unpublished results).

\section{Results}

No differences were found in the prevalence of smoking in the two areas at the start of the study in 1972.The finding at the end in 1977 was similar, although there were then fewer women smokers in North Karelia compared with the control area (table IIIm). Smoking among men decreased considerably in both areas; a smaller reduction occurred among women. There was a non-significant net reduction in North Karelia in the prevalence of smoking of $2.5 \%$ for men and $6.1 \%$ for women. When the reported amount of smoking was taken into account the net reduction among men in North Karelia $(9.8 \%)$ was significant (table IVm). This was a result of the finding that in 1972 North Karelian men smoked more than those in the control area, but this difference disappeared during the study. Otherwise the findings on the reported amount of smoking were similar to those on the prevalence of smoking.

Table Vm shows that at the start of the study the mean serum cholesterol concentrations were higher in people in North Karelia compared with the control area for all sex and age groups. During the study this difference was reduced among women and reversed among men. Thus the net reduction in North Karelia was highly significant among men $(4.1 \% ; 0.29 \mathrm{mmol} / 1(11.1 \mathrm{mg} / 100 \mathrm{ml}))$ and non-significant $(1.2 \% ; 0.08 \mathrm{mmol} / 1(3.0 \mathrm{mg} / 100 \mathrm{ml}))$ among women.

Relative weight, as measured by the Quetelet body mass index, was closely similar in both areas at the start of the study for both men $(25.7 v 25.6)$ and women (26.3 $v 26.2)$ and increased slightly in both areas during the study.

The mean casual systolic blood pressure in 1972 was somewhat higher in North Karelia than in the control area (table VIm). This difference was reversed during the follow-up period, so that the net reduction in North Karelia was highly significant for both men $(3.6 \% ; 5.3 \mathrm{~mm} \mathrm{Hg})$ and women $\left(4.8^{\circ} ; 7.2 \mathrm{~mm} \mathrm{Hg}\right)$. The mean casual diastolic blood pressure in men in 1972 was lower in North Karelia than in the control area, while virtually no difference was found among women (table VIIm). During the study a highly significant net reduction occurred in North Karelia in both men $(2.8 \% ; 2.6 \mathrm{~mm} \mathrm{Hg})$ and women $(3.7 \% ; 3.4 \mathrm{~mm} \mathrm{Hg})$. The main aim of the programme with regard to blood pressure was to reduce raised pressures. Thus it was necessary to ascertain the prevalence of raised values defined by the arbitrary criteria of casual blood pressure $\geqslant 175 \mathrm{~mm} \mathrm{Hg}$ systolic or $\geqslant 100 \mathrm{~mm} \mathrm{Hg}$ diastolic, or both. The prevalence of such raised values in 1972 was similar among men in the two areas but was higher among women in North Karelia than the control area. The net reduction in the prevalence of raised values in North Karelia was substantial and highly significant in both men $(43.5 \%$ ) and women $(48.5 \%)$ (table VIIIm).

The estimates of CHD risk, described above, showed that in 1972 the North Karelians had a higher mean score than the population in the control area (table IXm). During the follow-up period this difference was reversed among men and disappeared among women. Thus the net reduction in the estimated CHD risk in North Karelia was highly significant in the men $(17.4 \%)$ and the women $(11.5 \%)$. The net reduction among the men was greatest in the oldest and youngest age groups, and was considerably smaller in those aged 40-49 in 1972. Among women these findings were reversed, the net reduction being greatest in those aged 40-49.

\begin{tabular}{|c|c|c|c|c|c|c|c|c|}
\hline & \multicolumn{4}{|c|}{ Barcline survey } & \multicolumn{4}{|c|}{ Terminal survey } \\
\hline & \multicolumn{2}{|c|}{ North Karche } & \multicolumn{2}{|c|}{ Controt ares } & \multicolumn{2}{|c|}{ Nornh Katelis } & \multicolumn{2}{|c|}{ Control ares } \\
\hline & Men & Women & Men & Women & Men & women & Men & Wumen \\
\hline 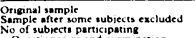 & $\begin{array}{l}2570 \\
2427 \\
2228\end{array}$ & 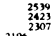 & $\begin{array}{l}3709 \\
3199 \\
3159\end{array}$ & 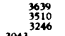 & 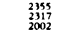 & $\begin{array}{l}\substack{2373 \\
\text { 2372 } \\
2121} \\
2121\end{array}$ & 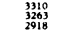 & 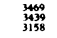 \\
\hline 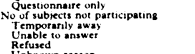 & 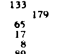 & $\begin{array}{l}n_{111} \\
31 \\
3 \\
3\end{array}$ & 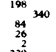 & $\begin{array}{l}203 \\
0604 \\
18 \\
19\end{array}$ & $\substack{35 \\
\text { s. } \\
23}^{315}$ & $1_{14}^{221}$ & $\underbrace{435}_{15}$ & $\begin{array}{l}51 \\
31 \\
32 \\
28\end{array}$ \\
\hline $\begin{array}{l}\text { Unthown reaton } \\
\text { Participetion rate }\end{array}$ & 89926 & & & 173925 & 227804 & 105906 & 200904 & 106 918 \\
\hline
\end{tabular}

$\mathrm{Im}$

\begin{tabular}{|c|c|c|c|c|c|c|c|c|}
\hline \multirow{3}{*}{ Are (years) } & \multicolumn{4}{|c|}{1972} & \multicolumn{4}{|c|}{1977} \\
\hline & \multicolumn{2}{|c|}{$\begin{array}{l}\text { North Karchio } \\
\end{array}$} & \multicolumn{2}{|c|}{ Control arces } & \multicolumn{2}{|c|}{ North Karchis } & \multicolumn{2}{|c|}{ Control ares } \\
\hline & $\begin{array}{c}\text { Participation } \\
\text { rate }\end{array}$ & $\begin{array}{c}\text { No } \\
\text { studied }\end{array}$ & $\begin{array}{c}\text { Participation } \\
\text { rate }\end{array}$ & $\begin{array}{c}\text { No } \\
\text { studicd }\end{array}$ & $\begin{array}{c}\text { Partcipatuon } \\
\text { rate }\end{array}$ & $\begin{array}{c}N_{0} \\
\text { studied }\end{array}$ & 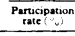 & $\begin{array}{c}\text { No } \\
\text { ttudied }\end{array}$ \\
\hline 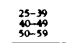 & $\begin{aligned} 909 \\
938 \\
930 \\
940\end{aligned}$ & $\begin{array}{l}9222 \\
509 \\
547\end{array}$ & $\begin{array}{l}8 \begin{array}{l}872 \\
932 \\
917\end{array} \\
917\end{array}$ & 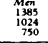 & $\begin{array}{c}885.5 \\
873 \\
870 \\
870\end{array}$ & $\begin{array}{l}9277 \\
6113 \\
402 \\
02\end{array}$ & 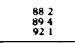 & $\begin{array}{l}\text { 1374 } \\
\text { s92 } \\
6522 \\
\end{array}$ \\
\hline Total & 926 & 2228 & 902 & 3159 & 804 & 2002 & 894 & 2918 \\
\hline $\begin{array}{r}25-39 \\
50-99 \\
50-59 \\
\end{array}$ & $\begin{array}{c}2010 \\
97010 \\
9551\end{array}$ & $\begin{array}{l}932 \\
716 \\
659 \\
659\end{array}$ & 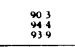 & 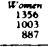 & $\begin{aligned} 9113 \\
915 \\
884 \\
884\end{aligned}$ & 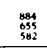 & $\begin{array}{l}910 \\
939 \\
914\end{array}$ & 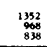 \\
\hline Total & 952 & 2307 & 925 & 3240 & 906 & 2121 & 918 & 3158 \\
\hline
\end{tabular}

IIIm

\begin{tabular}{|c|c|c|c|c|c|c|c|c|}
\hline \multirow{2}{*}{ A } & \multicolumn{3}{|c|}{1972} & \multicolumn{3}{|c|}{1997} & \multicolumn{2}{|c|}{ Net reduction in North Karelis } \\
\hline & $\begin{array}{l}\text { Norh } \\
\text { Karteis } \\
\end{array}$ & $\begin{array}{c}\text { Control } \\
\text { strea }\end{array}$ & Differennic & $\begin{array}{c}\text { North } \\
\text { Katctiot }\end{array}$ & $\begin{array}{c}\text { Control } \\
\text { onted } \\
\end{array}$ & Difference & Aboviute & $\%$ \\
\hline 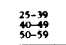 & 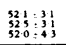 & 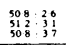 & $\begin{array}{r}12 \\
13 \\
12: 40 \\
12: 56\end{array}$ & 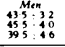 & 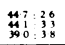 & 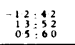 & 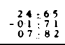 & 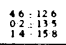 \\
\hline Tous & $522: 21$ & 509.1 .8 & 12.27 & 432:22 & $433: 18$ & 01.29 & 13.0 & $25: 70$ \\
\hline 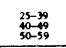 & $\begin{array}{r}15527 \\
11527 \\
66.19 \\
\end{array}$ & 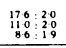 & 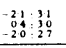 & 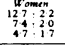 & 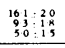 & 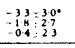 & 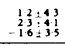 & $\begin{array}{r}70: 27.7 \\
199=359 \\
248: 53 \\
\end{array}$ \\
\hline Total & $117 \div 1.3$ & $131 \cdot 12$ & -16.18 & $89: 1.2$ & 111.11 & $-2.2: 16^{\circ}$ & $0.7+24$ & $01: 200$ \\
\hline
\end{tabular}

\begin{tabular}{|c|c|c|c|c|c|c|c|c|}
\hline \multirow{2}{*}{ 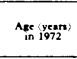 } & \multicolumn{3}{|c|}{1972} & \multicolumn{3}{|c|}{197} & \multicolumn{2}{|c|}{ Net reduction in North Karelia } \\
\hline & $\begin{array}{l}\text { Nornh } \\
\text { Karrtiat }\end{array}$ & 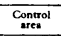 & Difference & $\begin{array}{l}\text { Norat } \\
\text { Karction }\end{array}$ & $\begin{array}{c}\text { Control } \\
\text { arca }\end{array}$ & Difference & Abrolute & $\because$ \\
\hline 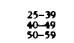 & 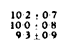 & $\begin{array}{l}9.500 .6 \\
9.070 .7 \\
7.7 \pm 0.7\end{array}$ & 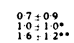 & 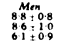 & $\begin{array}{l}8.9+0.96 \\
8.4+0.7 \\
61+0.707\end{array}$ & 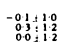 & 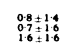 & $\begin{array}{c}79+134 \\
72+16 \\
109+102\end{array}$ \\
\hline Tonal & $9 \cdot 9: 04$ & $89=0.4$ & $10+0.0$ & $81 \pm 0.5$ & $81 \div 04$ & $0.0 \pm 07$ & $1.0 \pm 0.90$ & $9.8+9.0$ \\
\hline $\begin{array}{c}25-39 \\
\text { 25-39. } \\
50-59 \\
50-59\end{array}$ & $\begin{array}{l}1.60 .03 \\
0.370 .3 \\
07: 02\end{array}$ & 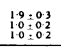 & $\begin{array}{r}-0.3=0.4 \\
-0.3 \\
-0.3 \geq 0.4\end{array}$ & 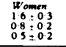 & $\begin{array}{l}20.0 .3 \\
10.03 \\
05 \pm 02\end{array}$ & 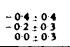 & 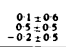 & 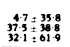 \\
\hline Total & $13 \pm 0.2$ & $1.4 \pm 0.1$ & $-0.1 \pm 0.2$ & $11 \neq 0.2$ & $13 \times 02$ & -02202 & $0.1 \pm 0.3$ & $8.0 \pm 249$ \\
\hline
\end{tabular}

$\mathrm{Vm}$

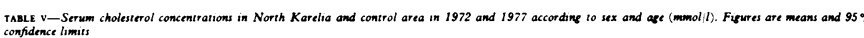

\begin{tabular}{|c|c|c|c|c|c|c|c|c|}
\hline \multirow[b]{2}{*}{$\begin{array}{l}\text { Agec veatr } \\
\text { in } 1972\end{array}$} & \multicolumn{3}{|c|}{1972} & \multicolumn{3}{|c|}{1977} & \multirow{2}{*}{\multicolumn{2}{|c|}{$\begin{array}{l}\text { Net reduction in North Karelie } \\
\text { Abrolutuc }\end{array}$}} \\
\hline & $\begin{array}{c}\text { Norn } \\
\text { Katrelia } \\
\end{array}$ & 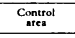 & Difference & $\begin{array}{l}\text { North } \\
\text { Karchia } \\
\end{array}$ & 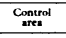 & Difference & & \\
\hline 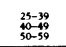 & 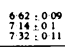 & 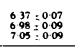 & 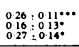 & 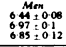 & 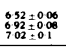 & 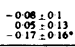 & 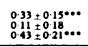 & 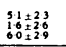 \\
\hline Total & $090: 006$ & $673 \div 0.05$ & $0.23 \times 0.07 \cdots$ & $67: 0.05$ & $6.75: 0.05$ & $-0.05: 0.07$ & $029 \pm 01 \cdots$ & $41+15$ \\
\hline 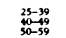 & 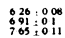 & 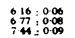 & 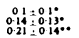 & 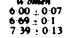 & 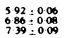 & 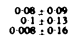 & 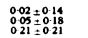 & $\begin{array}{l}0.3 \pm 22 \\
03 \pm 27 \\
27 \pm 28\end{array}$ \\
\hline Toual & $600 \div 000$ & $67: 0.05$ & $0.16 \pm 0.07 \cdots$ & $0.48=0.06$ & 661005 & $-008=000$ & $0.08=0.11$ & $12 \pm 10$ \\
\hline
\end{tabular}

VIm

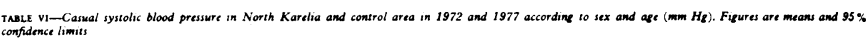

\begin{tabular}{|c|c|c|c|c|c|c|c|c|}
\hline \multirow{2}{*}{ 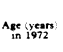 } & \multicolumn{3}{|c|}{1972} & \multicolumn{3}{|c|}{1977} & \multicolumn{2}{|c|}{ Net reduction in North Karethe } \\
\hline & 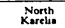 & $\begin{array}{c}\text { Control } \\
\text { tret }\end{array}$ & Difference & $\begin{array}{ll}\text { North } \\
\text { Karteta } \\
\end{array}$ & 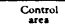 & Difference & Abrolute & $\because$ \\
\hline $\begin{array}{l}25-39 \\
\text { 25-39.9 } \\
50-59\end{array}$ & 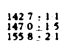 & 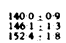 & $\begin{array}{l:ll}27 & 19 \\
09 & 19 \\
34 & 28\end{array}$ & 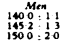 & 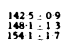 & 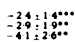 & 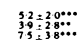 & 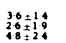 \\
\hline Total & $1973: 09$ & $1450: 07$ & $24: 11 \cdots$ & $1439: 08$ & $1468: 07$ & $-29+11 \cdots$ & $5: 3+16 \cdots$ & $3.6 \pm 1 \cdot 1$ \\
\hline $\begin{array}{l}25-39 \\
\text { 25-30 } \\
50-59 \\
50-59\end{array}$ & 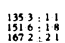 & $\begin{array}{l}1205: 09 \\
1652 \\
1630\end{array}$ & 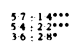 & 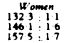 & 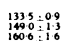 & 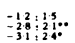 & 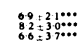 & $\begin{array}{l}5: 1+1.5 \\
3,4+2.0 \\
40 \pm 2.2\end{array}$ \\
\hline Total & $1694: 11$ & $1441 \div 09$ & $53+14 \cdots$ & $1435=09$ & $1454 \div 08$ & $-1.9+12^{* *}$ & $72 \pm 19 \cdots$ & $48+1 \cdot 3$ \\
\hline
\end{tabular}




\section{Discussion}

The aim of this study was to assess the changes occurring in the chosen primary CHD risk factors during the intensive community programme in North Karelia. The net reduction in risk factors in North Karelia (the reduction in North Karelia minus the reduction in the control area) was considered to be the effect of the programme. The neighbouring county of Kuopio was chosen as the control area because at the start of the study it most closely resembled North Karelia in terms of, for example, mortality and morbidity from CVD and geographical, occupational, economic, and social features. ${ }^{18}$ The results indicate that, in addition to the observed changes in North Karelia, some favourable changes also occurred in the control area. It is difficult to say whether these changes represent national trends, whether the project was already contributing to national trends (even though an attempt had been made to avoid this), or whether the programme in North Karelia had some spillover effects to the neighbouring county. A direct comparison with the national trends cannot be made owing to a lack of comparable surveys. On the other hand, the national trends are heavily influenced by changes in the big cities in southern Finland, which are likely to be very different from those in the less developed and more rural areas of eastern Finland.

We assessed the programme's effect on the risk factors by examining independent cross-sectional population samples in the two areas at the start and end of the programme. A longitudinal follow-up of the baseline survey samples, as was done, for example, in the Stanford three-community study, ${ }^{19}$ would have had advantages from the analytical point of view. On the other hand, this was considered to be inappropriate, because the subjects examined in the baseline survey were directly influenced by the epidemiological survey and did not represent the general community any more. ${ }^{20}$ Probably the change would look better than it actually was if judged by a follow-up of the same people. ${ }^{21}$

A further problem in evaluating the effect of the community programme was related to the age range of the sample populations. In planning the project we decided that the programme, although comprehensive, would particularly cover the middleaged male population, which had the highest rate of disease at

\section{MINIPRINT TABLES VII-IX}

\begin{tabular}{|c|c|c|c|c|c|c|c|c|}
\hline \multirow[b]{2}{*}{ A } & \multicolumn{3}{|c|}{1972} & \multicolumn{3}{|c|}{1977} & \multicolumn{2}{|c|}{ Net reduction in North Karelis } \\
\hline & $\begin{array}{l}\text { North } \\
\text { Karctia }\end{array}$ & $\begin{array}{c}\text { Control } \\
\text { serea }\end{array}$ & Difference & $\begin{array}{l}\text { Norh } \\
\text { Karcita } \\
\end{array}$ & $\begin{array}{c}\text { Control } \\
\text { artad }\end{array}$ & Difference & Abrolute & $\because$ \\
\hline 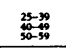 & 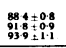 & 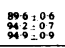 & 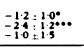 & 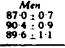 & 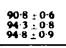 & 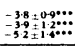 & 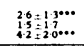 & 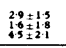 \\
\hline Toual & $908 \pm 0.5$ & $924: 04$ & $-1.5 \div 0.7 \cdots$ & $886=05$ & $928=04$ & $-4,1 \div 0,7 \cdots$ & $26 \pm 0.9 \cdots$ & $2.8 \pm 1.8$ \\
\hline 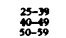 & 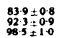 & 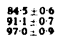 & 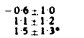 & 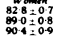 & 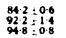 & 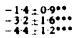 & 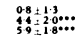 & $\begin{array}{l}1001+16 \\
19+2.2 \\
60 \pm 1.8\end{array}$ \\
\hline Toeal & $907 \pm 06$ & $90.0 \pm 0.4$ & $0.7 \pm 0.7$ & $86.8 \div 0.5$ & $80.5 \div 0.0$ & $-2.7 \div 0, \cdots \cdots$ & $3 \cdot 4+10^{2} \cdots$ & $37 \times 1.1$ \\
\hline
\end{tabular}

\begin{tabular}{|c|c|c|c|c|c|c|c|}
\hline \multirow[b]{2}{*}{ 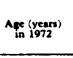 } & \multicolumn{3}{|c|}{1972} & \multicolumn{3}{|c|}{1977} & \multirow{2}{*}{$\begin{array}{l}\text { Ner exduction in North Karchia } \\
\text { Abololute }\end{array}$} \\
\hline & 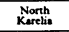 & $\begin{array}{c}\text { Contral } \\
\text { stert }\end{array}$ & $\begin{array}{l}\text { Difference } \\
\end{array}$ & $\begin{array}{c}\text { North } \\
\text { Kertath }\end{array}$ & 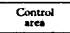 & 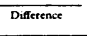 & \\
\hline 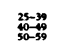 & 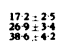 & 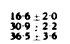 & 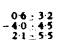 & 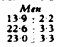 & 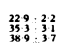 & 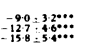 & 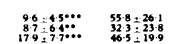 \\
\hline Toot & $236=19$ & 2060116 & $-0.4 \div 25$ & 187.17 & $303: 17$ & $-116: 24 \cdots \cdots$ & 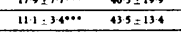 \\
\hline 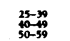 & 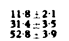 & 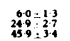 & 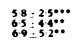 & 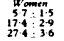 & 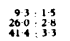 & 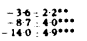 & 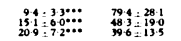 \\
\hline Toat & $200 \pm 19$ & $229: 1.5$ & $=24 \cdots$ & $152+15$ & $229 \pm 15$ & & \\
\hline
\end{tabular}

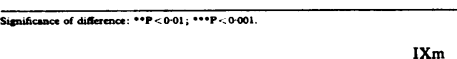

\begin{tabular}{|c|c|c|c|c|c|c|c|c|}
\hline \multirow[b]{2}{*}{ 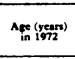 } & \multicolumn{3}{|c|}{1972} & \multicolumn{3}{|c|}{1977} & \multicolumn{2}{|c|}{ Ner redactor in Norh Karctat } \\
\hline & $\begin{array}{l}\text { Kornh } \\
\text { Korrat }\end{array}$ & $\begin{array}{c}\text { Comprol } \\
\text { stret }\end{array}$ & Difisetence & $\begin{array}{l}\text { Norath } \\
\text { Kortath }\end{array}$ & $\begin{array}{l}\text { Contred } \\
\text { surter }\end{array}$ & Differenone & Abonolute & $\because$ \\
\hline 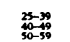 & 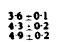 & 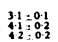 & 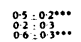 & 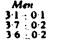 & 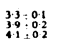 & 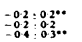 & $\begin{array}{l}87=0 \\
0 \\
0\end{array}$ & 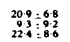 \\
\hline Toeat & $4.1 \pm 0.01$ & $37: 0.1$ & $0,0=02 \ldots$ & $34 \div 0.0$. & $37: 01$ & $-03.01 \cdots$ & $07,02 \cdots$ & $174 \div 48$ \\
\hline 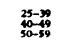 & 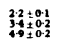 & 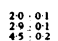 & 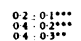 & 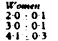 & 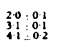 & 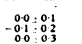 & 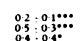 & 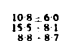 \\
\hline Trost & $\frac{1+102}{3 y \geq 0.1}$ & 30.02 & $04001 \cdots$ & 290.1 & $29-01$ & 0,0001 & $04=02^{2} \cdots$ & $115=52$ \\
\hline
\end{tabular}

the start of the study. ${ }^{2} 6$ Owing to the community nature of the programme, however, the age range of the samples in the baseline survey was broadened to comprise people aged 25-59 and both sexes. At the end of the five-year follow-up period another representative sample, then aged 30-64, was examined. This age range was chosen in order to assess the changes in the same age group, though not the same subjects. Using the same age cohorts increases the comparability of the baseline and terminal measurements, because if the sample at the end had had the same age range as that at the start the comparability would have been diminished owing to possible, unknown differences in the age cohorts in the two areas. The method used, however, means that the sample at the end was five years older, which is important in terms of the absolute changes in the two areas-that is, possible reductions in the risk factors could have been counterbalanced by an increase due to aging-but not in terms of the comparison with the development in the control area or the net reduction in North Karelia. As well as this aging, the age distribution and other background features of the samples were closely similar in 1972 and $1977,{ }^{18}$ and, for example, age standardisation did not change the results at all.

The measurements were carried out by specially trained nurses, who were carefully instructed before the surveys to use standardised and internationally accepted techniques for taking the measurements. Great efforts were made to carry out both surveys in exactly the same way in both areas. The time of year was also the same in the two areas and two surveys. Although different staff took the measurements in the two areas (especially measurements of blood pressure), they were recruited in a similar way, trained jointly, and treated similarly. The fact that several staff were used (12 in both surveys) minimised the effect of possible differences in technique in the comparisons between the two areas, as shown by subsequent analyses.

The serum samples were first deep frozen, and were sent to the laboratory for analysis in mixed order from the two areas. The same laboratory was used all the time; it is standardised against the international WHO reference laboratory, but the actual technique was changed between 1972 and $1977 . .^{1415}$

The validity of the results on smoking might have been reduced by incorrect self-reporting. A random subsample of subjects was given a second interview about smoking by the trained nurses, who were not aware of the answers in the questionnaire. Among the men the agreement between the two results was $93 \%$ when classified by an interval of five cigarettes, $97 \%$ when classified by an interval of 10 cigarettes, and $99 \%$ when classified as a smoker or non-smoker. The validity of these results on smoking was further confirmed by the results of serum thiocyanate determinations made during the terminal survey. Further analysis will also be carried out to establish any dietary changes that took place and might have contributed to the observed changes in cholesterol concentration.

The quality of the data is strengthened by the high participation rates in the two surveys and both areas. Probably some of the non-participants had moved from the area and should have been removed from the original sample as not belonging to the population any more. Thus the true "drop-out rate" is likely to be even smaller. The participation rate at the end of the study was slightly lower in North Karelia than the control area, which was probably due to the many examinations that were organised during the programme in North Karelia. We studied relatively large samples so that we could detect changes that might not be great in an individual but might be highly relevant when the mean values in the whole population were concerned.

The main approach in the preventive programme was a multifactorial one-that is, we wished to influence the combination of the risk factors. Thus after each risk factor had been assessed separately a CHD risk estimate was calculated and presented using a multiple logistic function (Walker-Duncan model). This approach was strengthened by the fact that the coefficients for this model had been derived from a five-year follow-up of a representative population sample in the same population. ${ }^{17}$ When the risk factors were assessed jointly by 
using the estimated overall CHD risk the net reduction in North Karelia was $17 \cdot 4 \%$ among men and $11.5 \%$ among women. The finding that the change was greater among men accords with our aim, which was to study and influence the male population in particular, because of its greater incidence of $\mathrm{CHD}$ and higher mortality. Apparently among men the programme most favourably influenced those in the older age group, who, possibly having become aware of their own personal risks, best complied with the programme. On the other hand, the results also suggest that the younger generation was eager to adopt a new life style.

Even if the two areas were comparable at the start of the study, the figures show that North Karelia often had slightly higher starting values. This is natural because of the historical background of the project: because North Karelia had the highest incidence of CVD the intervention programme was started there. As a whole, the programme seems to have reversed the differences between the two areas. Thus the favourable net change was partly caused by the levels of the risk factors in North Karelia catching up with those in the control area. This is in fact one way of looking at the entire effect of the programme in North Karelia: the county that was most backward in Finland-with regard not only to the CVD risk but also to socioeconomic and other features-through an intensive programme surpassed other areas in health development. ${ }^{22}$

When the risk factors are studied separately it is apparent that a highly significant net reduction in serum cholesterol concentration was achieved in the North Karelian population. Although the final results of the dietary changes are not yet available, the programme in North Karelia probably led to greater dietary changes relevant to reducing cholesterol concentrations than those in the control area. The hypertension programme also seems to have been successful, but its impact on the mean values in the population was reduced by the fact that it mainly aimed at reducing raised blood pressures. Thus the effect of the programme on reducing raised blood pressures was considerably greater than that on the mean blood pressures in the whole population.

The smallest net reduction occurred in the prevalence of smoking. This was because, despite a considerable reduction in smoking in North Karelia, smoking also decreased appreciably in the control area. There may be several explanations for this: the antismoking education in the neighbouring county may have influenced the control study. Also, especially towards the end of the period, national interest in antismoking activities increased, to some extent inspired by this project. The Finnish Parliament passed new antismoking legislation in 1976. This national activity may have influenced the control area just before the final survey. This possibility is supported by the findings of the internal follow-up surveys in North Karelia during the programme. They indicate that most of the reduction in smoking in North Karelia occurred at the beginning of the intervention after the first intensive public propaganda, and that during the rest of the period this lower level of smoking was maintained. ${ }^{23}$

As a whole, the results indicate a net reduction in the risk factors in North Karelia, which is considered to be the effect of the programme. Many of the changes are statistically highly significant and likely to be important from the point of view of the population, even if not from the point of view of an individual. There is no question of being able to change the risk factors in individual subjects. But can the risk factors be changed and by what means among whole populations or large areas, and ultimately among whole nations? Some reports indicate that this could be the case at least in some conditions. ${ }^{24-27}$ Furthermore, risk factors are often "spontaneously" changing for economic, commercial, health, or other reasons. ${ }^{28-32}$ On the other hand, many health education campaigns and programmes have had little or no permanent effects on actual health behaviour. ${ }^{33-37}$ Also, although national legislation is obviously powerful, in most countries it is restricted and depends greatly on changes in the population's attitude.

The results presented here indicate that the level of the risk factors did change in the whole population of North Karelia during the five-year programme. Five years is obviously long enough for the changes in individual subjects to be called permanent; another question, however, is how the changes at community level will proceed after this period. Further questions are, of course, what the reasons for this favourable change were and whether this study could be done elsewhere. We want to emphasise that a community programme like this is ultimately designed to test whether the given programme, which should be designed so that it can be applied on a larger scale, is feasible and effective in the given conditions. The relative contribution of the different components and the effect of the given conditions on the success can be evaluated to only a limited extent.

We think that in so far as this project is concerned the comprehensive community-based approach and integration of the activities into the social- and health-service structure of the community were particularly important factors. In other words, the aim was to change the community as a whole rather than individual subjects living in it. With hindsight it is easy to say that the conditions in North Karelia were favourable for the programme. At the planning stage, however, great concern was expressed because, for instance, the area was a rural one with a low socioeconomic standing, it had few medical resources, dairy farming was among the main sources of livelihood, unemployment was high, etc. At the beginning of the programme it was shown that the risk factors in question were clearly associated with rural living, less education, low income, etc. ${ }^{38}$ Despite all this, the programme was found feasible and effective. Further analyses of the available data will give more information about the changes in the risk factors among different subgroups of the population, about associated changes-for example, in health knowledge, attitudes, etc-and about changes related to the different parts of the programme. Thus, despite the limitations mentioned above, these results contribute much to our epidemiological knowledge of the CHD risk factors. Many severe limitations exist for arranging classical randomised trials to prove the causal role of these behaviour- and environmentrelated risk factors; many of these limitations can be avoided by using a community-based approach. ${ }^{39}$

The good feasibility of the programme in North Karelia has been reported elsewhere. ${ }^{114041}$ This report indicates that the community-based comprehensive programme was also effective at least with respect to its aim to reduce the levels of the given risk factors in the population. According to considerable evidence, this should subsequently lead to a reduction in mortality and morbidity from CVD. ${ }^{42-45}$ Whether this was the case during the five-year period and what indicators were concerned will be analysed and reported later. We intend also to continue monitoring these indicators for a longer period in order to detect the possible consequences of the programme for the future occurrence of these diseases.

Requests for reprints should be addressed to Professor Pekka Puska, co-ordinating centre of the North Karelia Project, University of Kuopio, Box 40, 70101 Kuopio 10, Finland.

\section{References}

${ }^{1}$ Keys, T, American Heart Association Monograph No 29. New York, American Heart Association, 1970.

2 Bolander, A-M, Statistical Report 1971. Stockholm, National Central Bureau of Statistics, 1971.

3 World Health Organisation, Public Health in Europe. Copenhagen, WHO/EURO, 1976.

${ }^{4}$ Ovcarov, V, and Bystrova, V, World Health Statistics Quarterly, 1978, 31, 208.

5 Puska, P, Suomen Lääkärilehti, 1972, 27, 3071.

- Puska, P, and Mustaniemi, H, Acta Medica Scandinavica, 1975, 197, 211.

${ }^{7}$ Reunanen, A, Kansaneläkelaitoksen fulkaisuja, Monograph No AL:8. Helsinki, Social Security Research Institution, 1977.

${ }^{8}$ Leppo, K, Lindgren, J, and Ritamies, M, in Yearbook of Population Research in Finland XII, 1971. Helsinki, Population Research Institute, 1972.

${ }^{9}$ Puska, P, Hallinto ja Terveys, 1977, 1, 4. 
10 Puska, P, WHO Chronicle, 1973, 27, 55.

11 Puska, P, Publications of the University of Kuopio, Community Health series $A: 1$. Kuopio, University of Kuopio, 1974.

12 Puska, $\mathrm{P}$, in The Promotion of Health: New Trends and Perspectives. Bethesda, Maryland, Van Nostrand Reinhold Co. In press.

${ }^{13}$ Rose, G, and Blackburn, H, Cardiovascular Survey Methods. Geneva, WHO, 1968.

${ }_{14}$ Technicon AutoAnalyzer Methodology N-77. Technicon, 1969.

${ }_{15}$ Carr, J, and Drekter, J, Clinical Chemistry, 1956, 2, 353.

16 Puska, P, Rimpelä, M, and Virtamo, J, Suomen Lääkärilehti, 1972, 27, 2950.

17 Walker, S, and Duncan, D, Biometrika, 1967, 54, 167.

18 Central Statistical Office of Finland, Living Conditions 1950-1975, Statistical Surveys, No 58. Helsinki, Central Statistical Office of Finland, 1977.

19 Farquhar, J, et al, Lancet, 1977, 1, 1195.

${ }^{20}$ Glasunov, I, et al, International fournal of Epidemiology, 1973, 2, 137.

21 Puska, P, et al, Acta Medica Scandinavica, 1978, 204, 381.

22 National Board of Health of Finland, Year Book of National Board of Health 1971-1973, Official Statistics of Finland XI:74. Helsinki, National Board of Health of Finland, 1978.

${ }^{23}$ Tuomilehto, J, et al, International fournal of Health Education, 1978, 21, suppl No 4.

24 World Health Organisation, The Prevention of Coronary Heart Diseases. Copenhagen, Regional Office for Europe, WHO, 1977.

${ }^{25}$ Maccoby, N, et al, fournal of Community Health, 1977, 2, 100.

${ }^{26}$ Schönberger, J, Abstracts of the VIII World Congress of Cardiology, Tokyo, 17-23 September 1978. Tokyo, International Society and Federation of Cardiology, 1978.

27 Tunstall Pedoe, $\mathrm{H}$, et al, Transactions of the European Society of Cardiology, 1978, $1,1$.

${ }_{28}$ Malmros, H, Acta Medica Scandinavica, 1950, 138, suppl.

${ }^{29}$ Levy, R, Preventive Medicine, 1978, 7, 464.
${ }^{30}$ Shimamoto, T, and Komachi, Y, Abstracts of the International Symposium on Essential Hypertension, Herzeg Novi, Yugoslavia, 23-27 October 1978 Belgrade, Federal Executive Council of the SFR Yugoslavia, 1978.

${ }^{31}$ Cooper, R, et al, Abstracts of the VIII World Congress of Cardiology, Tokyo, 17-23 September 1978. Tokyo, International Society and Federation of Cardiology, 1978.

${ }^{32}$ Rimpelä, M, Kansanterveystieteen Laitoksen fulkaisuja, series M:40/78. Tampere, University of Tampere, 1978.

${ }^{33}$ Hyman, H, and Sheatsky, P, Public Opinion Quarterly, 1947, 11, 412.

${ }^{34}$ O'Keefe, T, Public Opinion Quarterly, 1971, 31, 242.

${ }^{35}$ Robertson, L, et al, American fournal of Public Health, 1974, 64, 1071.

36 World Health Organisation, Report on a Working Group on Health Education in Community Control of Cardiovascular Diseases. Geneva, WHO, 1976.

${ }^{37}$ Banks, M, World Smoking and Health, 1978, 3, 39.

${ }^{38}$ Tuomilehto, J, et al, Preventive Medicine, 1978, 7, 539.

${ }^{39}$ Farquhar, J, American fournal of Epidemiology, 1978, 108, 103

${ }^{40}$ Tuomilehto, J, Publications of the University of Kuopio, Community Health, series $A: 2$. Kuopio, University of Kuopio, 1975.

${ }_{11}$ Puska, $\mathrm{P}$, et al, in Childhood Prevention of Atherosclerosis and Hypertension. New York, Raven Press. In press.

42 Report of Inter-Society Commission for Heart Disease, Circulation, 1970, 42, December.

43 World Health Organisation, Methodology of Multifactor Preventive Trials in Ischaemic Heart Disease. Copenhagen, Regional Office for Europe, WHO, 1973.

44 World Health Organisation, The Prevention and Control of Major Cardiovascular Diseases. Copenhagen, Regional Office for Europe, WHO, 1974.

45 Report of a Joint Working Party of the Royal College of Physicians of London and the British Cardiac Society, fournal of the Royal College of Physicians, 1976, 10, 213.

(Accepted 19 September 1979)

\section{Summary and conclusions}

A comprehensive community programme studying the control of cardiovascular diseases (CVD) was carried out in North Karelia, Finland, between 1972 and 1977. The main objective was to reduce the mortality and morbidity of CVD, particularly in middle-aged men. Changes in the mortality and incidence of CVD were monitored by community-based registers of cases of acute myocardial infarction (AMI) and stroke and data on death certificates. During the programme the total mortality in the area decreased by $5 \%$ and the mortality from CVD decreased by $13 \%$ among men and $31 \%$ among women aged 30-64 years. The incidence of AMI fell by $16 \%$ among men and $5 \%$ among women, while that of cerebral stroke fell by $\mathbf{3 8 \%}$ among men and $\mathbf{5 0 \%}$ among women. Changes in mortality in North Karelia

\footnotetext{
Co-ordinating Centre of the North Karelia Project: University of Kuopio, Box 40, 70101 Kuopio 10, Finland, and Epidemiological Research Unit, National Public Health Laboratory, Kuopio JUKKA T SALONEN, MD, special research investigator PEKKA PUSKA, MD, MA, director HARRI MUSTANIEMI, MD, senior physician
}

were compared with those in a matched control area; the difference between the two areas was not significant.

The true effect of the programme cannot be deduced from these results, but mortality from CVD and the incidence of AMI and stroke fell during the five years studied. Thus the changes in mortality and morbidity of CVD accorded with the initial objectives of the programme.

\section{Introduction}

Cardiovascular diseases (CVD) are the main cause of death in middle-aged people, especially men, in most industrialised countries. In many countries-for example, the USA and Finland-the most common manifestation is coronary heart disease (CHD), while elsewhere-for example, Japan-it is cerebrovascular disease. In the early 1970s it was quite obvious that the incidence of CVD varied considerably between countries. The age-standardised mortality from heart disease in men aged $35-64$ years varied from $466 \cdot 8 / 100000$ population in Finland to 73.3 in Japan, and the mortality from cerebrovascular diseases varied from $139 \cdot 6 / 100000$ in Japan to $34 \cdot 7$ in Denmark. ${ }^{1}$

When the World Health Organisation (WHO) started its systematic cardiovascular programme in the 1960 s one of the first tasks was to obtain a more reliable picture of the differences in mortality among countries. Were they true or possibly due only to different national diagnostic or coding practices? In 